\title{
Modification of a montmorillonite-illite clay using alkaline hydrothermal treatment and its application for the removal of aqueous $\mathrm{Cs}^{+}$ions
}

\author{
B. Öztop, T. Shahwan * \\ Department of Chemistry, Faculty of Science, Izmir Institute of Technology, 35430 Urla, Izmir, Turkey
}

Received 31 October 2005; accepted 2 December 2005

Available online 18 January 2006

\begin{abstract}
A montmorillonite-illite clay was modified using alkaline hydrothermal treatment (reflux method) and applied to the removal of aqueous Cs ${ }^{+}$ ions. The alkaline solutions were prepared by dissolving $\mathrm{NaOH}$ in seawater and in distilled water, and the effect of the two alkaline media on the sorption capacities of the modified clay was discussed. The modified materials were characterized using XRD, SEM/EDS, and FTIR. As a result of the modification, the original mineral was partially transformed into a zeolitic material with spherical morphology. The results showed that the modification improved the $\mathrm{Cs}^{+}$uptake capacity of the starting clay, with the clay modified in distilled water medium demonstrating higher sorption capacity. The sorption data were adequately described using the Freundlich and Dubinin-Radushkevich isotherm models.

(c) 2006 Elsevier Inc. All rights reserved.
\end{abstract}

Keywords: Montmorillonite; Illite; Alkaline hydrothermal treatment; Sorption; $\mathrm{Cs}^{+}$

\section{Introduction}

Montmorillonite and illite are among the best-known clay minerals, which are widely studied by many researchers as $\mathrm{Cs}^{+}$ sorbents (e.g., [1-6]). The alkaline hydrothermal treatment of montmorillonite is less common than that of kaolinite, where such treatment leads to the formation of hydrated feldspathoid or hydroxysodalite [7]. The aim of this treatment is to convert the layered (lamellar) structure of the clay into a porous structure composed of channels and cavities that can provide sorption locations for sorbate ions/molecules. During the hydrothermal alkaline treatment, ions such as $\mathrm{Na}^{+}$and $\mathrm{K}^{+}$act as templates around which the aluminosilicate units polymerize to produce large pores in the structure [8]. The morphology and grain size of the produced zeolitic material depend on the reaction conditions and the silica content of the starting material. This modification aimed at increasing the uptake capacity of the solid and obtaining products that can behave selectively toward a particular cation. Zeolitic material synthesized in seawater medium from bentonite using reflux and autoclave methods was reported to show selectivity toward $\mathrm{NH}_{4}^{+}$ions in seawater [8].

\footnotetext{
* Corresponding author. Fax: +90 2327507509.

E-mail address: talalshahwan@iyte.edu.tr (T. Shahwan).
}

Cesium is important from a radioactive waste viewpoint. It has several radioactive isotopes, the most important of which are ${ }^{134} \mathrm{Cs}\left(t_{1 / 2}=2.06\right.$ years $),{ }^{135} \mathrm{Cs}\left(t_{1 / 2}=3.0 \times 10^{6}\right.$ years $)$, and ${ }^{137} \mathrm{Cs}\left(t_{1 / 2}=30.17\right.$ years), produced in nuclear fission. Due to their long half-lives, both ${ }^{135} \mathrm{Cs}$ and ${ }^{137} \mathrm{Cs}$ are principal radiocontaminants.

In this study, hydrothermal alkaline treatment was performed using the reflux method. Seawater and distilled water were used in the preparation of the alkaline solutions and their effect on the modified clay was discussed. The produced material was characterized using X-ray diffraction (XRD), scanning electron microscopy (SEM), energy dispersive X-ray spectroscopy (EDS), and infrared spectroscopy (FTIR). The synthesized solids were then tested as sorbents for $\mathrm{Cs}^{+}$ions and their uptake capacity was compared with that of the starting montmorillonite-illite clay. The concentrations of both ions in the filtrates following the sorption experiments were determined using atomic absorption spectroscopy (AAS).

\section{Experimental}

The clay mineral used in this study was obtained from Aldrich (Cat. 28,152-2). The alkaline solutions were prepared with a concentration of $3.75 \mathrm{M} \mathrm{NaOH}$ dissolved in seawater, 
obtained from the Bay of Urla town situated on the Aegean coast of Turkey. A sample of $15 \mathrm{~g}$ of the clay was then added to each solution and the mixtures were heated in an oil-bath up to the boiling point under reflux with continuous stirring for $8 \mathrm{~h}$. The suspensions were then left for an aging period of $16 \mathrm{~h}$ in dry and dark medium. The samples were finally filtered, washed with deionized water, and oven-dried. The produced powders were then characterized and used in the sorption experiments. The experiments were repeated by applying alkaline solutions in which $\mathrm{NaOH}$ was dissolved in distilled water.

The sorption experiments were performed using the batch method. Kinetic studies were carried out at concentrations of $5 \times 10^{-3}$ and $1 \times 10^{-4} \mathrm{M}$ of $\mathrm{CsCl}$ over mixing periods ranging from $10 \mathrm{~min}$ up to $48 \mathrm{~h}$ in order to reveal the time required for equilibrium. To study the effect of loading on the sorption process, 10 -ml aliquots of $5 \times 10^{-3}, 1 \times 10^{-3}, 5 \times 10^{-4}$, and $1 \times 10^{-4} \mathrm{M} \mathrm{CsCl}$ solutions were mixed with $0.1 \mathrm{~g}$ of the solids using a magnetic stirrer for $24 \mathrm{~h}$ at $25^{\circ} \mathrm{C}$. The solid phases were finally separated from the liquid phases by centrifugation. The filtrate was then analyzed by flame AAS using a thermal elemental SOLAAR M6 Series-type instrument.

The FTIR spectra were recorded using a Nicole Magna 550 type instrument in the range $400-4000 \mathrm{~cm}^{-1}$. The samples were introduced as pellets of powders diluted with $\mathrm{KBr}$ powder. The resolution was $4 \mathrm{~cm}^{-1}$ and a total of 32 scans were recorded for each spectrum. The software used to process the results was Omnic 1.3. XRPD analysis of the mineral phases was performed using a Philips X'Pert Pro diffractometer. The samples were first ground, mounted on holders, and then introduced for analysis. The source consisted of $\mathrm{Cu} K \alpha$ radiation $(\lambda=1.54 \AA)$. Each sample was scanned within a $2 \theta$ range of $2^{\circ}-60^{\circ}$. The step size was 0.020 with a time per step duration of $60 \mathrm{~s}$. SEM/EDS characterization was carried out using a Philips XL-30S FEG type instrument. Prior to analysis, the solid samples were sprinkled onto adhesive carbon tapes supported on metallic disks.
Images of the sample surfaces were then recorded at different magnifications using SEM. The samples analyzed by EDS were sputter-coated with a thin conductive layer of gold to minimize the effect of charging arising from the application of higher voltages $(10-15 \mathrm{kV})$. EDS elemental analysis was performed at several randomly chosen points on the surface, and EDS mapping analysis was carried out at a magnification of $60 \times 65 \mu \mathrm{m}$.

\section{Results and discussion}

The clay fractions applied in this study were first characterized as received. The XRPD diagram of the montmorilloniteillite (MI) clay, shown in Fig. 1a, revealed that it contained primarily montmorillonite and illite, in addition to some quartz. The 001 feature of montmorillonite looks very broad, probably reflecting the amorphous nature of the mineral. The FTIR spectrum of the clay presented in Fig. 2a indicated the presence of a variety of aluminosilicate bands. The features at 3625 and $3440 \mathrm{~cm}^{-1}$ stem from the stretching vibrations of AlAl-OH and $\mathrm{H}$-bonded water in the clay structure, respectively. The band at $1046 \mathrm{~cm}^{-1}$ is referred to the stretching vibrations of the $\mathrm{Si}-\mathrm{O}$ bond, and the one at $915 \mathrm{~cm}^{-1}$ to the AlAlOH bending mode. $\mathrm{SiOAl}$ and SiOSi bending modes are reflected in the features appearing at 522 and $470 \mathrm{~cm}^{-1}$, respectively. The variations in these bands upon modification of the mineral are discussed later in this text. On elemental basis, the EDS analysis revealed that the percentage composition of MI is $62.47 \mathrm{O}, 25.71 \mathrm{Si}, 7.01$ $\mathrm{Al}, 2.36 \mathrm{Fe}, 1.09 \mathrm{Mg}, 0.65 \mathrm{Na}, 0.58 \mathrm{~K}$, and $0.27 \mathrm{Ca}$. According to SEM images, the particles of the mineral look like massive aggregates of irregular shapes, of sizes that amounts to several micrometers.

Upon modification of the clay, serious changes in its $\mathrm{Na}$ and Si content were observed. The EDS results showing the composition (as oxides) of MI clay and the one modified in seawater medium (MIS) are given in Table 1. The alteration of the min-

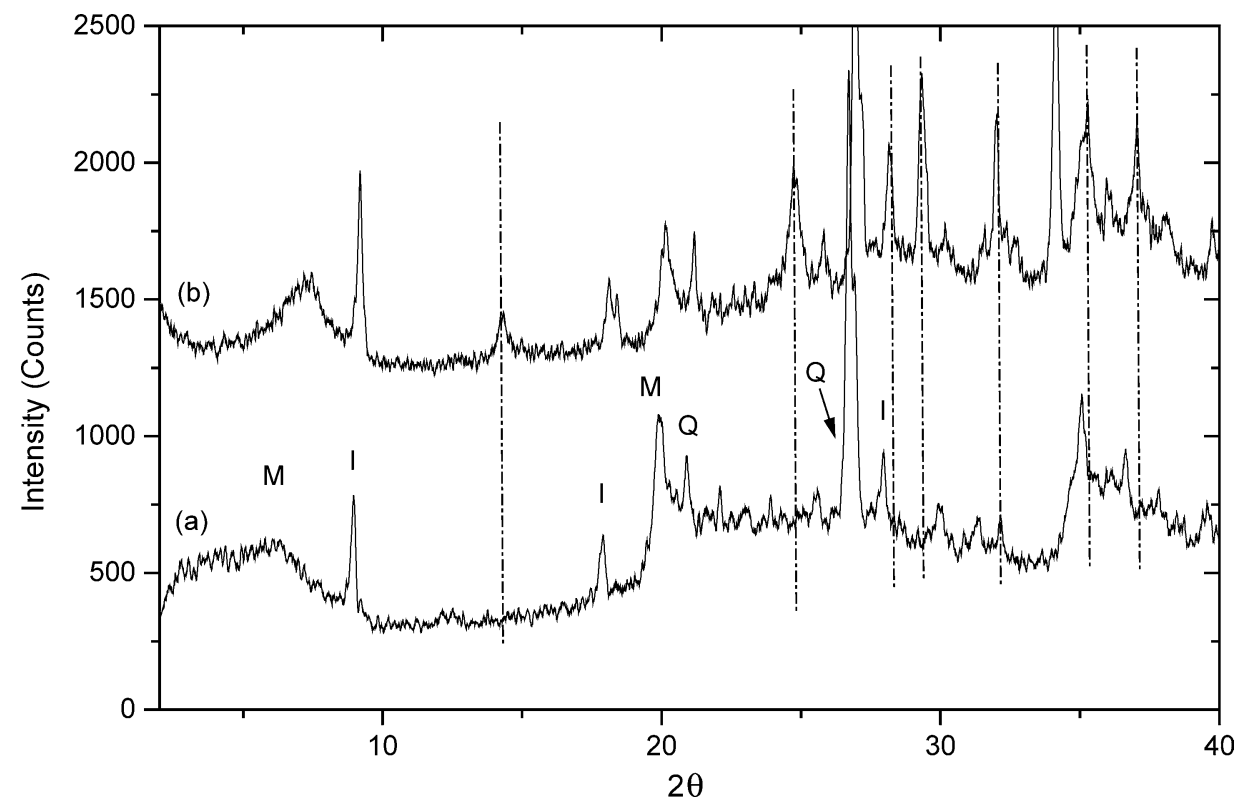

Fig. 1. The XRPD patterns of (a) MI, (b) MIS. M: montmorillonite, I: illite, Q: quartz. 


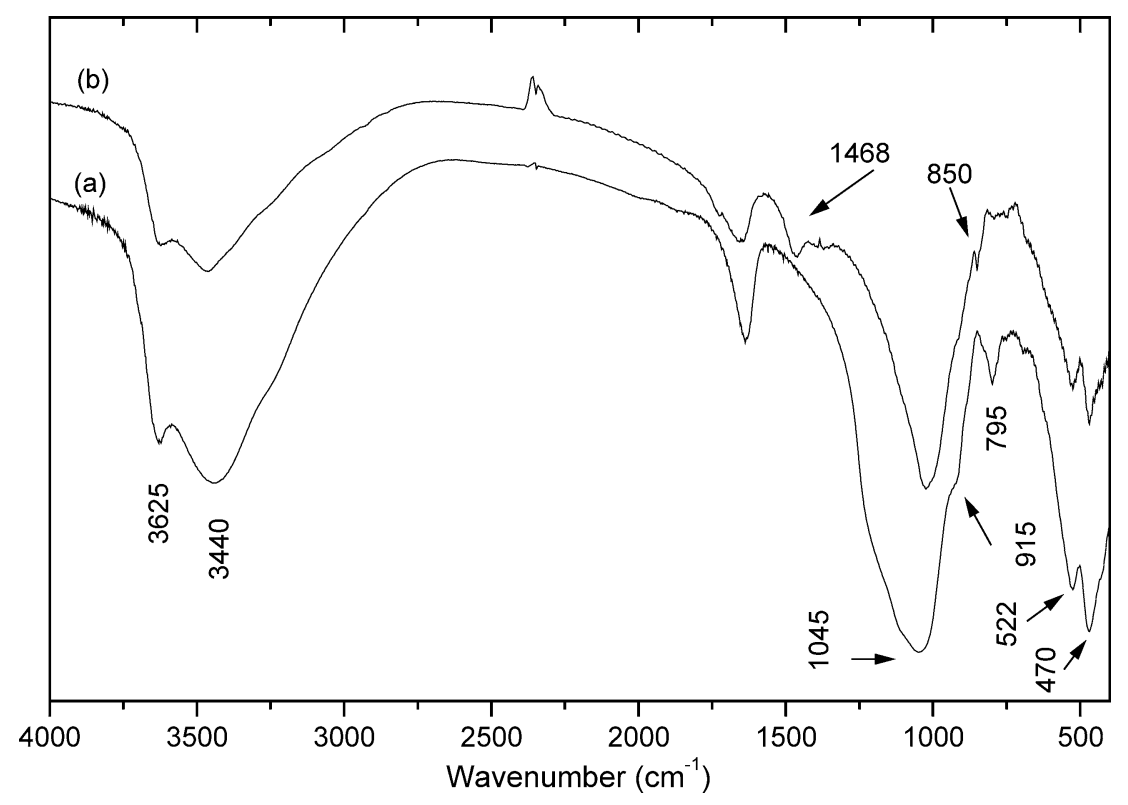

Fig. 2. FTIR spectra of (a) MI, (b) MIS.

Table 1

Oxide ratios of elements in MI, MIS, and MID

\begin{tabular}{llll}
\hline Ratio & MI & MIS & MID \\
\hline $\mathrm{SiO}_{2} / \mathrm{Al}_{2} \mathrm{O}_{3}$ & 7.32 & 4.01 & 3.30 \\
$\mathrm{Fe}_{2} \mathrm{O}_{3} / \mathrm{Al}_{2} \mathrm{O}_{3}$ & 0.34 & 0.41 & 0.42 \\
$\mathrm{MgO} / \mathrm{Al}_{2} \mathrm{O}_{3}$ & 0.31 & 0.62 & 0.33 \\
$\mathrm{CaO} / \mathrm{Al}_{2} \mathrm{O}_{3}$ & 0.04 & 0.08 & 0.07 \\
$\mathrm{Na}_{2} \mathrm{O} / \mathrm{Al}_{2} \mathrm{O}_{3}$ & 0.09 & 2.31 & 1.75 \\
$\mathrm{~K}_{2} \mathrm{O} / \mathrm{Al}_{2} \mathrm{O}_{3}$ & 0.08 & 0.11 & 0.12 \\
\hline
\end{tabular}

Note. All data are obtained from the EDS measurements.

eral is evident from the sharp changes in the $\mathrm{SiO}_{2} / \mathrm{Al}_{2} \mathrm{O}_{3}$ and $\mathrm{Na}_{2} \mathrm{O} / \mathrm{Al}_{2} \mathrm{O}_{3}$ ratios. Less significant changes are observed in the ratios of other oxides. The XRPD patterns are compared in Fig. 1. The figure reveals a partial transformation of the starting MI clay upon alkaline hydrothermal treatment. The fact that this treatment requires a high ratio of solution to solid to give high volume yields (product weight/reaction volume) is reported to be responsible for the limited conversion of the starting mineral into a zeolitic mineral [9]. The new features of the XRPD pattern of MIS (marked with dashed lines) apparently stem from more than a single type of zeolitic structures, as our search results did not yield a particular type of zeolite that could match all the reflections at once. Although a precise identification of all the peaks was difficult, the primary peaks with reflections at $2 \theta$ values of $14.28^{\circ}, 24.8^{\circ}, 34.19^{\circ}$, and $43.30^{\circ}$, corresponding to $d(\AA)$ values of $6.20,3.59,2.62$, and 2.09 , correspond to sodalite octahydrate-type zeolite.

The FTIR spectra given in Fig. 2 confirm a loss of intensity in the bands of the starting clay upon alkaline hydrothermal treatment and the development of new bands at 850 and $1463 \mathrm{~cm}^{-1}$ in the MIS spectrum. Typical SEM micrographs of MI and MIS are given in Figs. 3a and 3b. Fig. 3b shows part of the mineral structure in which a transformation into a spheroid granule morphology took place with a particle size that ranged approximately from 0.5 to $1 \mu \mathrm{m}$. It was early re-

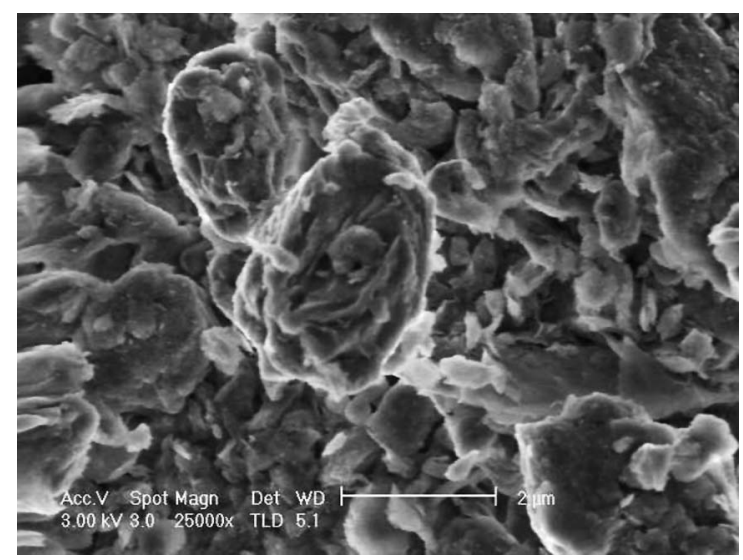

(a)

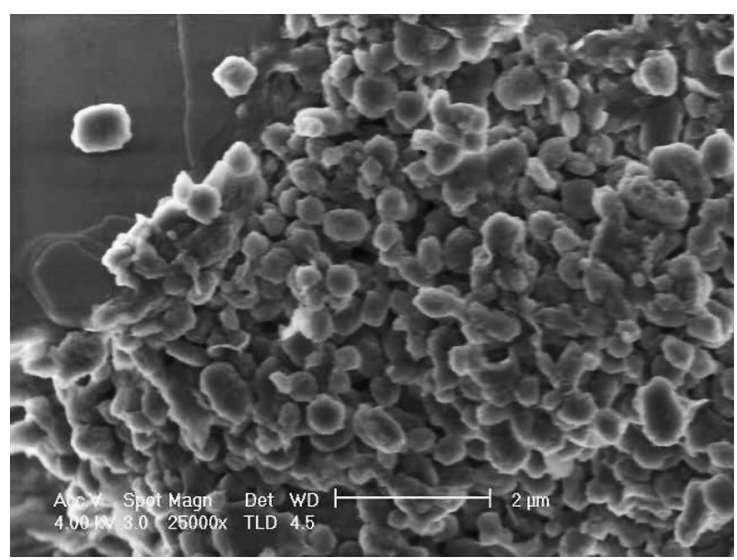

(b)

Fig. 3. Typical SEM micrographs of (a) MI, (b) MIS

ported that one of the factors that affect the morphology of the produced material in a zeolitization process is the characteristic of the medium, and that ions such as $\mathrm{Na}^{+}$and $\mathrm{K}^{+}$act as templates around which the aluminosilicate units polymerize to produce large pores in the structure [8]. 


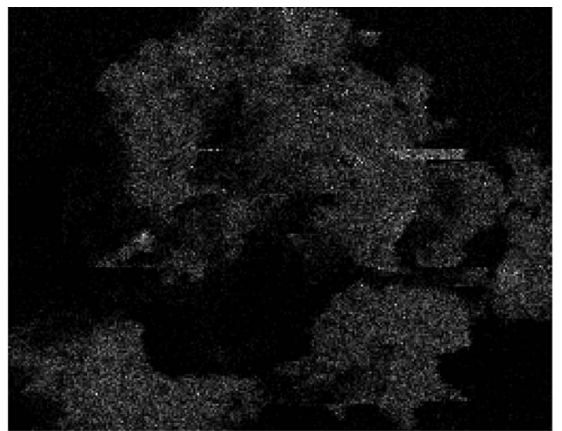

Al (K-line)

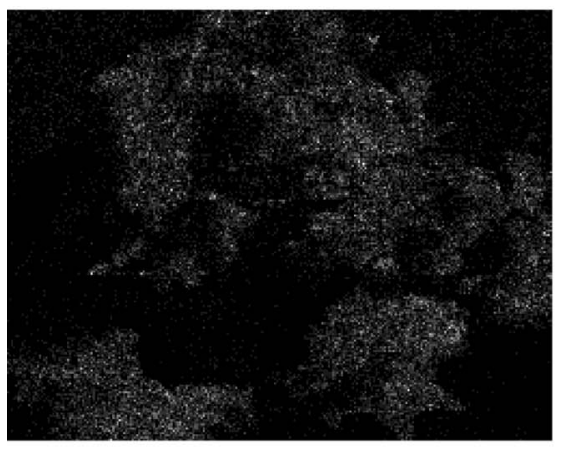

$\mathrm{Na}(\mathrm{K}-\mathrm{line})$

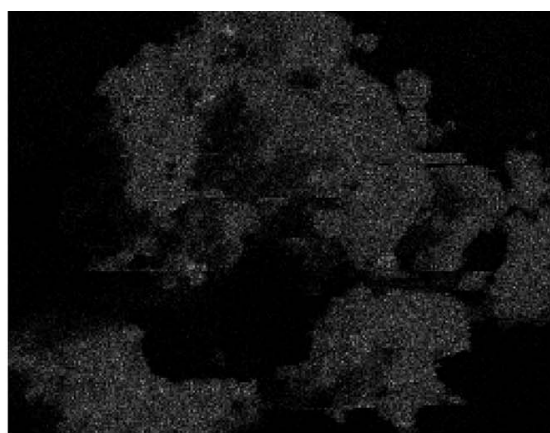

$\mathrm{Si}(\mathrm{K}-\mathrm{line})$

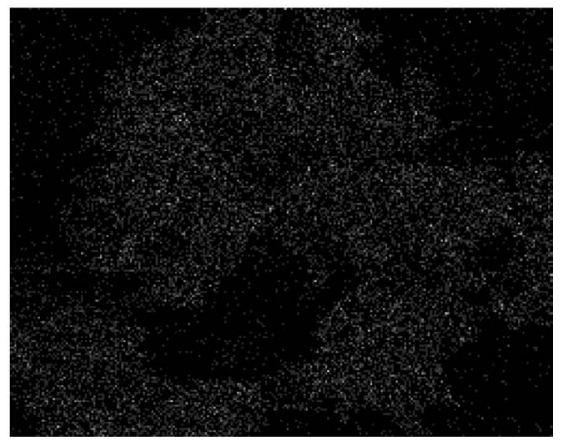

Cs (L-line)

Fig. 4. An EDS map showing the distribution of Al, Si, Na, and Cs on the surface of Cs-sorbed MIS.

The effect of $\mathrm{Cs}^{+}$sorption on the clay prior to and following modification was first examined using EDS. Multiple measurements on the surfaces of the minerals exposed to a $0.1 \mathrm{M}$ concentration of $\mathrm{Cs}^{+}$showed that the atomic percentage of this element on MI clay ranged from 1 to 1.5. In the case of sorption on MIS, however, the atomic percentage of Cs increased approximately by one-third, amounting to a value of about 2 . The EDS map of Cs on part of the solid surface rich with zeolited particles is shown in Fig. 4. The figure shows as well the distribution of $\mathrm{Na}, \mathrm{Al}$, and $\mathrm{Si}$ and corresponds to an area of $60 \times 65 \mu \mathrm{m}$. The maps demonstrate the association of $\mathrm{Na}$ with $\mathrm{Al}$ and $\mathrm{Si}$ in the zeolited fractions, but it is not possible to conclude what fraction of $\mathrm{Na}$ is structural and/or simply attached to the mineral surface. In general, a sharp decrease in the Na content of MIS was observed upon exposure to $\mathrm{Cs}^{+}$solutions. This large drop suggested that only a small fraction of the $\mathrm{Na}^{+}$ions available in the aqueous alkaline medium (originating from both seawater and $\mathrm{NaOH}$ ) are occupying skeletal positions in the modified clay, with the remaining larger fraction being loosely bound to the structure of the modified solid. This sharp drop in Na content was also verified by the intense characteristic $\mathrm{Na}$ signals upon analysis of the filtrate of the sorption experiments with atomic emission spectroscopy (AES).

The modification experiments were repeated by applying distilled water as a substitute for seawater. Characterization of the resulting new materials (MID) produced XRPD and FTIR features that largely resemble those of MIS. From the oxide compositions given in Table 1, it can be seen that replacing seawater with distilled water led to a decrease in the $\mathrm{Na}$ content of the modified clay. An important difference was also implied by the decrease in the number of particles possessing spheroid granular morphology. The MID solid was then applied as a sorbent for aqueous $\mathrm{Cs}^{+}$ions and its performance was compared with that of MIS. Preliminary results based on EDS measurements indicated that applying distilled water as a substitute for seawater in alkaline hydrothermal treatment yielded an important further increase in the sorption capacity of the synthesized material for $\mathrm{Cs}^{+}$ions. Typical EDS spectra of Cs-sorbed MI, MIS, and MID are shown in Fig. 5. It must be stressed that, in general, EDS results should be handled with care due to the errors that can arise because of inherent factors (poor detection ability for elements with atomic percentages below 5), in addition to possible anomalies that might originate from surface heterogeneity. Nevertheless, the conclusions drawn above were also confirmed by studying the sorption of $\mathrm{Cs}^{+}$on MI, MIS, and MID at multiple concentrations and analyzing the filtrates using AAS as shown below.

Preliminary kinetic studies performed at concentrations of $5 \times 10^{-3}$ and $1 \times 10^{-4} \mathrm{M}$ over mixing periods ranging from $10 \mathrm{~min}$ up to $48 \mathrm{~h}$ revealed that equilibrium was achieved within the first $2 \mathrm{~h}$ of mixing and that the equilibrium concentration of $\mathrm{Cs}^{+}$did not change over the remaining period. Based on this, the mixing period was fixed at $24 \mathrm{~h}$ in further experiments intended to investigate the effect of concentration (at initial values of $5 \times 10^{-3}, 1 \times 10^{-3}, 5 \times 10^{-4}$, and $1 \times 10^{-4} \mathrm{M}$ ) on the extent of retention of $\mathrm{Cs}^{+}$by MI, MIS, and MID. Following sorption, the concentration of aqueous $\mathrm{Cs}^{+}$ions was measured using AAS. The concentration of sorbed ions on the solid phases, $\mathrm{meq} / \mathrm{g}$, was then calculated from

$[C]_{\mathrm{s}}=\left([C]_{\mathrm{o}}-[C]_{1}\right)(V / M)$. 


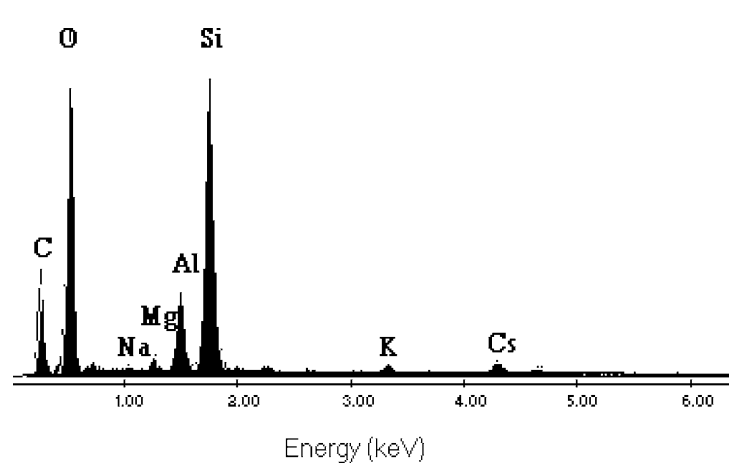

(a)

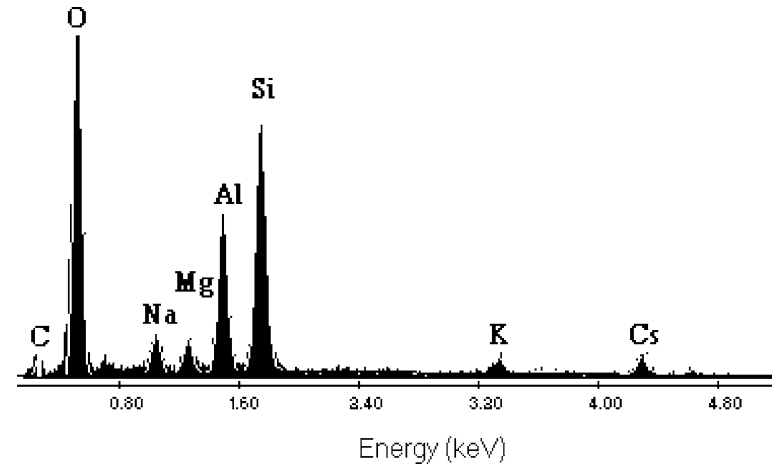

(b)

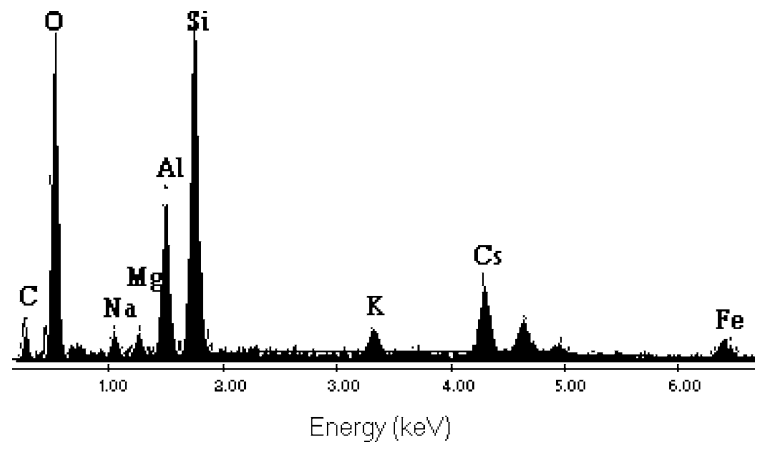

(c)

Fig. 5. Typical EDS spectra of Cs-sorbed minerals: (a) MI, (b) MIS, (c) MID.

Table 2

Percentage sorption of $\mathrm{Cs}^{+}$ions on MI, MIS, and MID at various loading values

\begin{tabular}{llll}
\hline Initial conc., M & MI & MIS & MID \\
\hline $5 \times 10^{-3}$ & 34 & 46 & 78 \\
$1 \times 10^{-3}$ & 44 & 65 & 89 \\
$5 \times 10^{-4}$ & 74 & 75 & 95 \\
$1 \times 10^{-4}$ & 86 & 92 & 97 \\
\hline
\end{tabular}

Here $[C]_{\mathrm{o}}$ is the initial concentration $(\mathrm{meq} / \mathrm{ml}),[C]_{1}$ is the concentration in the liquid at time $t(\mathrm{meq} / \mathrm{ml}), V$ is the volume of the solution (L), and $M$ is the mass of the solid (g). The percentage sorption (PS) was calculated utilizing the formula

$\mathrm{PS}=\frac{[C]_{\mathrm{o}}-[C]_{1}}{[C]_{\mathrm{o}}} \times 100 \%$.

The obtained values of PS are given in Table 2. The values clearly indicate that the sorption capacity of the minerals increases as MI < MIS < MID at all the studied concentrations.

The sorption data were fitted to the sorption isotherm models. The results showed that sorption can be best described using the Freundlich isotherm model and the Dubinin-Radushkevich (D-R) model; the formulas are given by

$$
\begin{aligned}
& {[C]_{\mathrm{s}}=k[C]_{1}^{n},} \\
& {[C]_{\mathrm{s}}=C_{\mathrm{m}} \exp \left(-K \varepsilon^{2}\right),}
\end{aligned}
$$

where $[C]_{\mathrm{s}}$ is the equilibrium concentration of the ion on the solid $(\mathrm{meq} / \mathrm{ml}),[C]_{1}$ is the equilibrium concentration of that ion in the liquid phase, and $n$ and $k$ are the Freundlich constants. In the $\mathrm{D}-\mathrm{R}$ isotherm, $\varepsilon$ is given by $R T \ln \left(1+1 /[C]_{1}\right)$, $R$ is the ideal gas constant $(8.3145 \mathrm{~J} / \mathrm{mol} \mathrm{K}), T$ is the absolute
Table 3

Freundlich constants $n, k$ and D-R constants $C_{\mathrm{m}}(\mathrm{meq} / \mathrm{g})$ and the sorption energy $E(\mathrm{~kJ} / \mathrm{mol})$, obtained from the sorption data of $\mathrm{Cs}^{+}$ions

\begin{tabular}{llclr}
\hline Solid & $n$ & $k$ & $C_{\mathrm{m}}$ & \multicolumn{1}{c}{$E$} \\
\hline MI & 0.52 & 2.95 & 0.36 & 10.1 \\
MIS & 0.62 & 12.0 & 0.79 & 9.6 \\
MID & 0.69 & 63.1 & 2.20 & 9.5 \\
\hline
\end{tabular}

temperature $(\mathrm{K})$, and $C_{\mathrm{m}}$ and $K$ are constants. The constants $n$, $k$ and $K, C_{\mathrm{m}}$ are obtained from the least-squares fits of the sorption data constructed using the linear forms of Eqs. (3) and (4). Freundlich and D-R plots are given in Fig. 6. The values of $n$, $k$, and $C_{\mathrm{m}}$ are all provided in Table 3 . The closer the value of $n$ to unity, the closer will be the sorption process to linear behavior. Linear sorption implies no change in the energetic barrier against sorption as coverage is increased or decreased, or in other words, that no significant change in the extent of sorbed amount occurs when the initial concentration of the sorbate is changed. The value of the Freundlich constant, $k$ (meq/g), can be correlated with the capacity of a particular solid for sorption under the particular experimental conditions. However, since the Freundlich isotherm does not predict a maximum coverage for a given sorbent, it is hard to say that $k$ corresponds to the maximum sorption capacity. The value of $k$ can, nevertheless, be useful in providing a qualitative comparison for the fixation affinity of a given sorbent toward different sorbates. On the other hand, the D-R constant, $C_{\mathrm{m}}(\mathrm{meq} / \mathrm{g})$, can be used as an estimate of the coverage capacity of the solid surface. From Table 3, it is evident that the linearity of sorption and sorption capacity increase in the order MI < MIS < MID. 

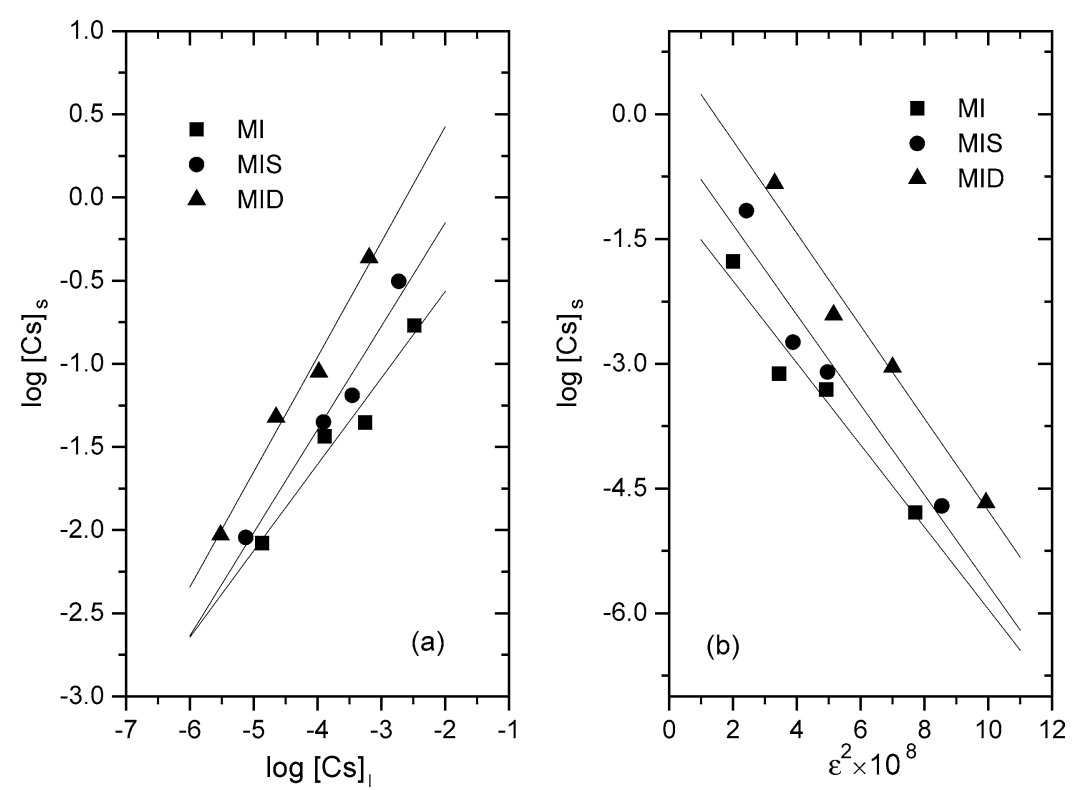

Fig. 6. Isotherm plots of $\mathrm{Cs}^{+}$sorption data: (a) Freundlich and (b) D-R isotherm.

The D-R constant $K$, obtained from the slope of the D-R plots, is related to the sorption energy, $E$, through

$E=\frac{1}{\sqrt{-2 K}}$.

Here $E(\mathrm{~kJ} / \mathrm{mol})$ refers to the amount of energy required to transfer one mole of sorbed ions from infinity in solution to the solid surface. The $E$ values are also included in Table 3 . The values are indicative that electrostatic types of forces are operative between the sorbate and the sorbent sites. The same conclusion can be drawn also from the values of $\Delta G^{\circ}$ of sorption presented in Table 4 and calculated using the equation

$\Delta G^{\circ}=-R T \ln R_{\mathrm{d}}$.

The table provides also the corresponding values of the distribution ratio, $R_{\mathrm{d}}(\mathrm{ml} / \mathrm{g})$, which represents the ratio $[\mathrm{Cs}]_{\mathrm{S}} /[\mathrm{Cs}]_{1}$, and is approximated here as an observed equilibrium constant that is valid at a particular initial concentration. The negative sign of the $\Delta G^{\circ}$ values is referred to the spontaneous nature of the sorption process. It is reported that if the sorption energy lies in the range $8-16 \mathrm{~kJ} / \mathrm{mol}$ then an ion-exchange mechanism will be dominant [10]. Earlier studies have discussed the large contribution of ion exchange in the sorption processes of cesium ions on montmorillonite and illite minerals (e.g., $[3,6])$, suggesting that external sites, as well as frayed edge sites (interlayer sites exposed along the edges of the clay platelets [6]), are involved in the exchange process. In a geological mixture containing montmorillonite and illite, both fractions are expected to take place in the exchange process, but the contribution of montmorillonite is reported to be more than two orders of magnitude larger than that of illite [11]. As outlined previously, the modification process results in a partial transformation of the lamellar structure into a porous one of zeolitic nature. Such a transformation could possibly affect the kinetic aspects of the
Table 4

Values of $R_{\mathrm{d}}(\mathrm{ml} / \mathrm{g})$ and $\Delta G^{\circ}(\mathrm{kJ} / \mathrm{mol})$ of sorption for $\mathrm{Cs}^{+}$retention by MI, MIS, and MID at various initial concentrations

\begin{tabular}{|c|c|c|c|c|c|c|}
\hline \multirow[t]{2}{*}{ Initial conc., $\mathrm{M}$} & \multicolumn{2}{|l|}{ MI } & \multicolumn{2}{|l|}{ MIS } & \multicolumn{2}{|l|}{ MID } \\
\hline & $R_{\mathrm{d}}$ & $\Delta G^{\circ}$ & $R_{\mathrm{d}}$ & $\Delta G^{\circ}$ & $R_{\mathrm{d}}$ & $\Delta G^{\circ}$ \\
\hline $5 \times 10^{-3}$ & 51 & -9.7 & 167 & -12.7 & 669 & -16.1 \\
\hline $1 \times 10^{-3}$ & 79 & -10.8 & 184 & -12.9 & 858 & -16.7 \\
\hline $5 \times 10^{-4}$ & 284 & -14.0 & 296 & -14.1 & 2074 & -18.9 \\
\hline $1 \times 10^{-4}$ & 617 & -17.6 & 1200 & -17.6 & 3133 & -19.9 \\
\hline
\end{tabular}

sorption process (extent of external diffusion versus pore diffusion), the elaboration of which necessitates a separate detailed study. Since the modification process involves introducing of a large amount of $\mathrm{Na}^{+}$ions into the mineral structure, with only part of these ions occupying skeletal positions, as outlined before, the reaction in which $\mathrm{Na}^{+}$is exchanged for $\mathrm{Cs}^{+}$in the modified mineral is expected to constitute a large part of the cation exchange process.

\section{Conclusions}

Alkaline hydrothermal treatment suffers mainly from the disadvantage of requiring large amounts of $\mathrm{NaOH}$ and the achievement of only partial conversion (under reflux) of the starting montmorillonite-illite clay. However, this treatment offers a means by which the sorption capacity of the clay toward $\mathrm{Cs}^{+}$is improved further. Within the studied range of $\mathrm{Cs}^{+}$concentration, the treatment process carried out using distilled water as a substitute for seawater is seen to be more effective in enhancing the sorption capacity of the zeolited material. The sorption data of $\mathrm{Cs}^{+}$on the three minerals MI, MIS, and MID was adequately described by Freundlich and D-R adsorption models. Further studies are being carried out to test the effect of the modification process on the uptake capacities of these solids toward other cationic sorbates. 


\section{Acknowledgments}

The authors thank Sinan Yilmaz for his help in the AAS measurements, and the Center of Material Research/Izmir Institute of Technology for their assistance in the SEM/EDS and XRPD measurements.

\section{References}

[1] T. Shahwan, H.N. Erten, J. Radioanal. Nucl. Chem. 253 (2002) 115.

[2] J. Vejsada, D. Hradil, Z. Řanda, E. Jelínek, K. Štulík, Appl. Clay Sci. 30 (2005) 53.
[3] C. Poinssot, B. Baeyens, M.H. Bradbury, Geochim. Cosmochim. Acta 63 (1999) 3217.

[4] S. Staunton, P. Levacic, J. Environ. Radioactiv. 45 (1999) 161.

[5] C. Willms, Z. Li, L. Allen, C.V. Evans, Appl. Clay Sci. 25 (2004) 125.

[6] J.L. Krumhansl, P.V. Brady, H.L. Anderson, J. Contam. Hydrol. 47 (2001) 233.

[7] A. Baccouche, E. Srasra, E. El Maaoui, Appl. Clay Sci. 13 (1998) 255.

[8] R. Ruiz, C. Blanco, C. Pesquern, F. Gonzalez, I. Benito, J.L. Lopez, Appl. Clay Sci. 12 (1997) 73.

[9] M. Park, C.L. Choi, W.T. Lim, M.C. Kim, J. Choi, N.H. Heo, Micropor. Mesopor. Mater. 37 (2000) 81.

[10] F. Helferrich, Ion Exchange, McGraw-Hill, New York, 1962.

[11] J. Kónya, N.M. Nagy, Z. Nemes, J. Colloid Interface Sci. 290 (2005) 350 . 